in diesem Falle in bezug auf $t$ ein noch günstigeres Resultat als im Falle der drei absoluten parallaktischen Winkel. Nachdem so bei nur 3 Sternen mittlerer Zenitdistanz für Zeit wie Polhöhe eine Genauigkeit von nur wenigen Bogensekunden erreicht worden ist, dürfte hiermit der Beweis erbracht sein, daß die photographische Methode allen visuellen Methoden mit Instrumenten gleicher Dimensionen völlig gleichwertig, ihnen an Einfachheit der instrumentellen Hilfsmittel und an der Art und Kürze der Ausführung, die übrigens z. B. auf Forschungsreisen auch astronomisch nicht vorgebildeten Beobachtern möglich ist, überlegen ist. Ferner hat die Methode den Vorteil, daß ihre Ergebnisse in jedem Moment nachgeprüft werden können, was speziell wieder für die Kritik von Forschungsreisen von erheblicher $\mathrm{Be}$ deutung sein kann. Eine Erhöhung der Genauigkeit ist nun einmal dadurch möglich, daß man eine größere Gruppe von Bildpunkten als 5 zu Anfang und Ende der Exposition heranzieht und auch eine stärkere Vergrößerung als 30 , als

Kiel, r 9 I 3 Febr. ro. mir bei dem Toepferschen Meßapparat zur Verfügung stand, benutzt. Ferner erhöht sich die Genauigkeit von selbst, wenn man der Platte eine größere Dimension gibt, als grade dem Objektivdurchmesser zukommt, und die Sterne dann längere Spuren beschreiben läßt, wobei etwa zu befürchtende unregelmäßige Distorsionswirkung bei einem besseren Objektive nicht zu erwarten ist, solange die Spurlänge nicht wieder zu groß gewählt wird. Da man ebenso mit einem Objektive größerer Öffnung oder längerer Brennweite als bei dem von mir benutzten Objektive den Sternspuren eine größere Länge geben kann, so wird man auch auf diesem Wege eine höhere Genauigkeit, als mir möglich war, erreichen und damit der photographischen Methode eine höhere Genauigkeit als den visuellen Methoden zuweisen können. Schließlich erhöht auch die zulässige Wahl viel geringerer Zenitdistanzen als bei meinen Aufnahmen, weil die Fehler im parallaktischen Winkel nur mit $\sin z$ multipliziert in Zeit und Polhöhe eingehen, von selbst die Genauigkeit des Resultats.

\title{
Observations de planètes
}

à I'Observatoire de Nice (Equatorial de $0.76 \mathrm{~m}$ d'ouverture) par $S$. Favelle.

\begin{tabular}{l|l|l|l|l|l|l|l|l|l|}
\hline Ig r 2 & T.m.deNice & $\Delta \alpha$ & $\Delta \delta$ & Cp. & $\alpha$ app. & $\log p \cdot \Delta$ & $\delta$ app. & $\log p \cdot \Delta \mid \operatorname{Red.~ad~l.~app.~} *$ \\
\hline
\end{tabular}

$108 \mathrm{Hecuba}$.

Oct. $\quad 4\left|10^{\mathrm{h}} 34^{\mathrm{m}} 24^{\mathrm{s}}\right|+2^{\mathrm{m}_{1}} 17^{\mathrm{s}} \cdot 43 \mid-3^{\prime} 12^{\prime \prime} \cdot 9$ \begin{tabular}{r|rrr|rr|rr}
5 & I I & 19 & 48 & +2 & 21.79 & -1 & 6.2 \\
9 & I I & 33 & 0 & + I & 31.54 & +4 & 33.2
\end{tabular}

\begin{tabular}{r|rrr|rr|rr|}
9 & I I & 33 & 0 & + I & 31.54 & +4 & 33.2 \\
I 5 & I I & I 5 & I 2 & + I & 52.55 & -2 & 28.0
\end{tabular}

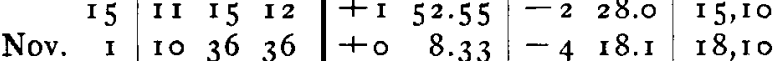

15,10
15,10
14,10
15,10
18,10

$\mathrm{I}^{\mathrm{h}} 30^{\mathrm{m}} 25^{\mathrm{s}} .97\left|9.350_{\mathrm{n}}\right|+\mathrm{I}^{\circ} 39^{\prime} 44^{\prime \prime} \cdot 6$

r $2940.96 \mid 9.135 n+r 23635.3$

I $26 \quad 43.0988 .890_{n}+122340.1$

I $22 \quad 9.7 \times \mid 8.764 n+12 \quad 2 \quad 46.5$

I $945.86|8.309|$ + Io $59 \quad 59.2$

76 Freia.

\begin{tabular}{rr|lll|ll|lr} 
Oct. & 9 & 9 & 37 & 48 & to & 32.63 & + 1 & 3.9 \\
& I 5 & 9 & 38 & 15 & to & 41.46 & -1 & 10.9 \\
I6 & 9 & 36 & 31 & +o & 11.57 & -4 & 18.8
\end{tabular} \begin{tabular}{l|l|l}
$\mathbf{1} 6,10$ & 2 \\
20,10 & 23 \\
18,10 & 23
\end{tabular}

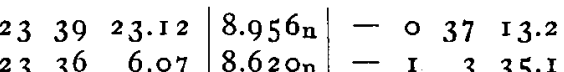
$233^{6} \quad 6.07 \mid 8.620_{n}-1 \quad 335.1$

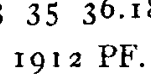

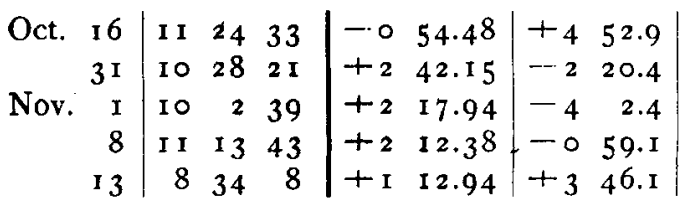
\begin{tabular}{r|}
20,10 \\
15,10 \\
15,10 \\
6,4 \\
14,10
\end{tabular} \begin{tabular}{lll|l|}
23 & 44 & 36.92 & 9.172 \\
23 & 36 & 21.03 & 9.227 \\
23 & 35 & 56.81 & 9.117 \\
23 & 33 & 35.75 & 9.470 \\
23 & 32 & 33.40 & 8.777
\end{tabular} + I I 2054.6 + I0 5 I 10.2 $+104928.2$ + 103855.8 $+103328.9$ 7 I $\mathrm{Marmula}$.

\begin{tabular}{ll|rrr|rr|rr|} 
Oct. & I 5 & I I & 53 & 8 & -0 & 49.85 & -5 & I 8.2 \\
I6 & I 2 & 45 & 27 & +3 & 38.47 & -4 & 5.7 \\
1 8 & I 3 & 0 & 36 & -0 & 22.32 & -8 & 35.3
\end{tabular} 20,10
14,10
16,10 $\circ 48 \quad 19.48|8.889|+94359.7$ \begin{tabular}{l}
$047 \mathrm{I} 4.59$ \\
\hline
\end{tabular} o 45 I2.5 I $|9.348|+93458.5$ \begin{tabular}{l|l|l}
0.675 & $+3.29+23.2$ & I \\
0.664 & $+3 \cdot 30+23 \cdot 3$ & 2 \\
0.662 & $+3.34+23.6$ & 3 \\
0.665 & $+3.39+24.0$ & 4 \\
0.677 & $+3.45+24.7$ & 5
\end{tabular} 9 I Aegina.

\begin{tabular}{rl|rrr|rr|rr|r|} 
Nov. 2 I & 8 & 56 & 26 & +0 & 8.62 & -1 & 52.0 & I 8, 10 \\
22 & 8 & 48 & 31 & -0 & 49.56 & -4 & 55.4 & 20,10 \\
25 & 9 & 3 & 19 & +1 & 30.09 & -7 & 8.6 & 15, 10
\end{tabular} 3 I 6 2 I 30 \begin{tabular}{lll|l|lll}
3 & 15 & 23.13 & $9.423 n$ & +20 & 45 & 16.4
\end{tabular} 3 I $31.16|9.329 n|+203^{6} \quad 5.2$ \begin{tabular}{l|l|l}
0.790 & $+3.28+20.6$ & 6 \\
0.785 & $+3.26+20.3$ & 7 \\
0.786 & $+3.26+20.3$ & 7
\end{tabular} 7 . . 


\begin{tabular}{|c|c|c|c|c|}
\hline Date & T.m.de Nice & $d \alpha$ & $d \delta$ & Cp. \\
\hline \multicolumn{5}{|c|}{ I $912-13$} \\
\hline Déc. I 3 & I I ${ }^{\mathrm{h}} \quad 2^{\mathrm{m}} 18^{\mathrm{s}}$ & $-2^{\mathrm{ml}} 47^{\mathrm{s}} \cdot 19$ & $-z^{\prime} 3$ I.". & I 5,10 \\
\hline I 4 & $9352 \mathrm{I}$ & -321.13 & - I 33.2 & 15,10 \\
\hline Janv. 4 & 101750 & -o 6.75 & -2 I 5.4 & I 8, I 0 \\
\hline 8 & I I 359 & +143.27 & - I 4.9 & I $5, \mathrm{IO}$ \\
\hline 12 & ro 728 & +310.55 & +329.2 & I 5,10 \\
\hline I 3 & $9 \quad 1326$ & $-425 \cdot 4^{2}$ & +458.7 & I 5,10 \\
\hline
\end{tabular}

$\alpha$ app. $|\log p \cdot \Delta| \quad \delta$ app.

$|\log p \cdot \Delta|$ Red. ad l. app. $\mid *$

1912

\begin{tabular}{ll|lll|ll|ll|l|} 
Déc. I 3 & I 2 & 17 & $3 \mathrm{I}$ & -0 & 53.18 & -5 & 3 I.I & I 2, I 2 \\
& I 4 & Io & 18 & I0 & - I & 46.84 & -4 & I 7.0 & I 4,I O
\end{tabular}

I 9 I $2 \mathrm{PZ}$

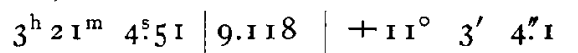
\begin{tabular}{lll|llll}
3 & 20 & 30.57 & $8.355 n$ & + I I & 4 & I.8
\end{tabular}

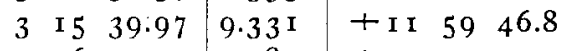
\begin{tabular}{lll|llll}
3 & 16 & 25.77 & 9.487 & +12 & I 7 & 3.3
\end{tabular}

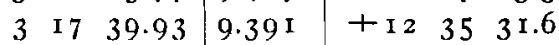

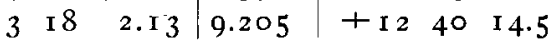

\section{9 I 2 PY.}

343 I $0.04|9.4 \times 5|+3244$ I I.0 342 I $6.38|8.282|+324525.2$ 200 Dynamene.

I 9 I 3

\begin{tabular}{r|rrr|rr|rr|r|} 
Févr. I I & I0 & 34 & 7 & - I & 0.79 & -0 & 45.1 & I 5, I0 \\
I 2 & I0 & 23 & 6 & - I & 54.39 & -0 & 28.3 & I 5, I0 \\
I3 & I0 & 0 & 23 & +0 & 59.01 & + I & 22.7 & I 5, I0 \\
24 & I I & I 2 & 57 & + I & 24.70 & -5 & 6.5 & I 5, I0 \\
25 & I0 & I9 & 20 & +0 & 49.3 & -5 & 54.8 & 20,10
\end{tabular}

\begin{tabular}{|l|l|l}
$0.68 \mathrm{I}$ & $+4.04+21.1$ & $2 \mathrm{I}$ \\
0.676 & $+4.04+21.0$ & $2 \mathrm{I}$ \\
$0.68 \mathrm{I}$ & $+0.67+7.7$ & 22 \\
0.702 & $+0.62+7.6$ & 23 \\
0.698 & $+0.60+7.5$ & 24 \\
0.666 & $+0.65+7.6$ & 25
\end{tabular}

$0.333|+4.63+22.9| 26$ 0.1 $75|+4.63+23.0| 26$

I 9 I 3

\begin{tabular}{l|lll|ll|ll|l|lll|l|l|lll|} 
Mars I I & II & I 6 & 47 & -2 & 58.83 & I I $_{2}$ & 26.6 & 14, IO & I0 & 5 & 18.3 I & 8.737 & +23 & 16 & 25.7
\end{tabular}

\begin{tabular}{rrr|l|rrr|r|r|r|r}
8 & 36 & 53.08 & $8.867 \mathrm{n}$ & $+2 \mathrm{I}$ & 7 & 9.7 & 0.532 & $+\mathrm{I} .89+\mathrm{r} .0$ & 27 \\
8 & 35 & 59.48 & $8.935 \mathrm{n}$ & $+2 \mathrm{I}$ & 7 & 26.6 & 0.534 & $+\mathrm{I} .89+\mathrm{I} . \mathrm{I}$ & 27 \\
8 & 35 & 6.92 & $9.083 \mathrm{n}$ & $+2 \mathrm{I}$ & 7 & 38.6 & 0.539 & $+1.89+\mathrm{I} .3$ & 28 \\
8 & 26 & 43.86 & 9.093 & $+2 \mathrm{I}$ & 3 & 54.0 & 0.540 & $+\mathrm{I} .85+2.2$ & 29 \\
8 & 26 & 8.47 & $8.4 \mathrm{I} 4$ & $+2 \mathrm{I}$ & 3 & 5.7 & 0.529 & $+\mathrm{r} .85+2.2$ & 29
\end{tabular}

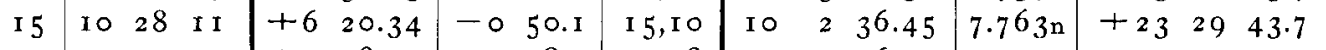

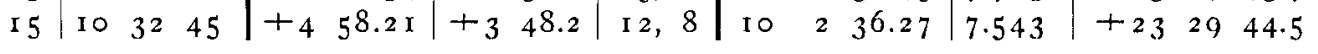

$0.508|+2.14-3.8| 30$ $0.479+2.1 \mathrm{I}-2.7 \quad 3 \mathrm{I}$ I 9 I 3 I 9 I 3 QU.

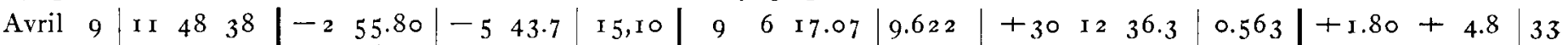

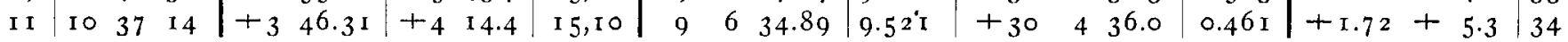
I 9 I 3 I 9 I $3 \mathrm{QV}$.

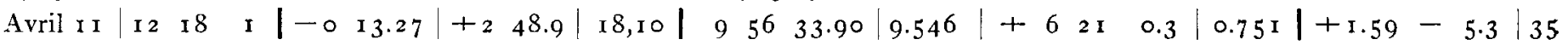

Positions moyennes des étoiles de comparaison.

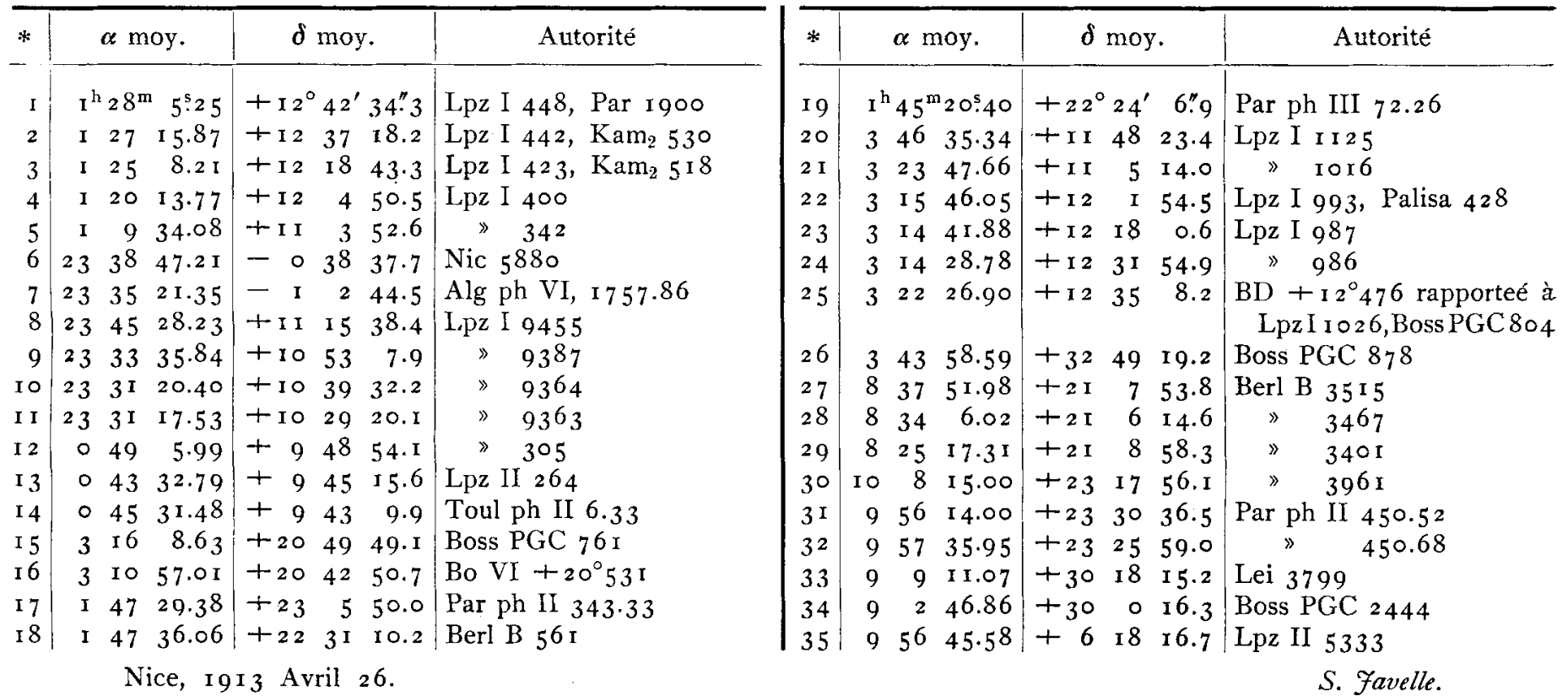

Inhalt zu Nr. 4660. A. Wilkens. Neue Prinzipe und Methoden zur geographischen Ortsbestimmung. 49. - S. Favelle. Observations de planètes. 93. 